\title{
Image Converting into Complex Networks : Scale- Level Segmentation Approach
}

\author{
Andrey Trufanov, Nikolay Kinash \\ Dept. of Mechanical Engineering \\ Irkutsk National Research Technical University \\ Irkutsk, Russia \\ troufan@gmail.com
}

\author{
Alexei Tikhomirov \\ Dept. of Global e-governance \\ Inha University \\ Incheon, Republic of Korea
}

\author{
Olga Berestneva \\ Institute of Cybernetics \\ National Research Tomsk Polytechnic University \\ Tomsk, Russia
}

\author{
Alessandra Rossodivita \\ Medical and University Center \\ Hospital Sacco \\ Milan, Italy
}

\begin{abstract}
Image analysis and recognition is being a contemporary domain for successful tries to apply complex networks as an instrument for thorough studies. Researchers noted that an image having traditionally converted into a network (i.e. taking into account Euclidean distance between pixels only) possesses nodes with similar number of admissible links and the concomitant graph demonstrates a regular topology. As a rule, pixel intensity difference is considered to escape regularity and reach complex property of the network. Contrary revealing more specific traits of an image current study proposes scale segmentation views -local, medium and global for an image to build a genuine complex network. Case study with two sample images manifests how the scales are connected with formation of a network topology.
\end{abstract}

Keywords-images, complex networks, conversion, topology, segmentation, spatial scales

\section{INTRODUCTION}

Since 1999 marked with enormous paper [1], there has been a boomed interest in network research, with the span from the analysis of small systems to the consideration of massive intricate multi-domain compositions. The latter stimulate being described by complex networks in their exaggerated forms of interdependent [2,3], multiplex[4-6], and combined structures [7], and some with severely dynamic properties [8].

One should emphasize that the role of network topology is of great importance as it stipulates functional specificities inherent to the system. Thus, dense structured social networks promotes conditions for rumor and infection spread, improper layout of railway station and tracks might enhance vulnerability and instability of transportation. [9].

Remarkebly, it was shown by [10] that even cities from specific continents tend to have particular topological properties.
Dealing with topological characteristics the complex networks connect the structure and the functions of the represented entity. It should be noted that network science grew up as a new scientific domain with original and effective scopes and instruments to describe and model diverse sophisticated systems. Unlike traditional graph theory as a monodiscipline with its simple structures and intricate language; the topologies covered by network science usually spring from complex systems. Those are often on interdisciplinary base, comprising a huge number of elements and their relationships. Another language accent indicates such a difference between network science and graph theory.

As a network spirit is in the center of many anthropogenic, technogenic and natural systems experts from diverse domains have successfully used complex networks to model the complex effects in socio-economic [11], ecological $[12,13$.$] ,$ biomedical [ 14,15$]$, information and communication $[16,17]$, cognitive [18], and technological spheres [19].

\section{RELATED WORKS}

One might find out that several papers are devoted to conversion of diverse data into networks, for example [18, 20-22]. However, there have been so far few works which treat images as complex networks in problems of image analysis and recognition [23,24].

Usually an image is interpreted as a complex network wherein each pixel represents a node. Nodes are linked or not according to proximity of location and color intensity between two pixels. It was emphasized [25] that in such an approach each node has same number of links and the network presents a regular behavior. Naturally, a structure with regular topology cannot be considered as a complex network, and so it is problematic to extract any relevant property and perform comprehensive analysis of images. To accomplish a behavioral exploration of the network the authors [25] analyzed derived graphs. They called such analysis as $\delta$ 
transformations. In each transformation a threshold $\boldsymbol{t}$ in the set of edges within the network graph was defined, in order to select its subset, so that each edge of the subset has the weight less or equal to $t$. For the series of $t=t_{1}, t_{2}, \ldots t_{n}$ new subgraphs were constructed on the base of the initial graph. These were proposed to treat as the samples of the complex network.

The paper [26] elaborated a network model which analyzes documents within a multi-scale context. In the proposed model a mesoscopic representation of texts was considered, where a node represented a large context, e.g. a set of adjacent sentences or paragraphs. More specifically, in their approach each node corresponded to several subsequent paragraphs.

The work [27] was probably the first which proposed to group pixels into "superpixels". Now superpixel techniques are often used as preprocessing steps of diverse computer vision tasks to make optimal their calculation phases in image segmentation per se, object recognition, classification and tracking [28].

In the paper [29] the authors implemented the technique of super pixels, to compress the cardinality of the set of network nodes. The work demonstrated that the technique gave good results both in accuracy and processing times on the examples of synthetic and real pictures.

In [30] an image was transformed into superpixel segments which included similar pixels and form compact regions.

\section{METHODOLOGY}

Close to the spirit of [31]

image partition and pertinent 3 spatial scales are taken into consideration. The increasing spatial scales, as zoom-out levels, are categorized as local, proximal ( medium), and global ones. The meaning of those is following ( Fig 1):

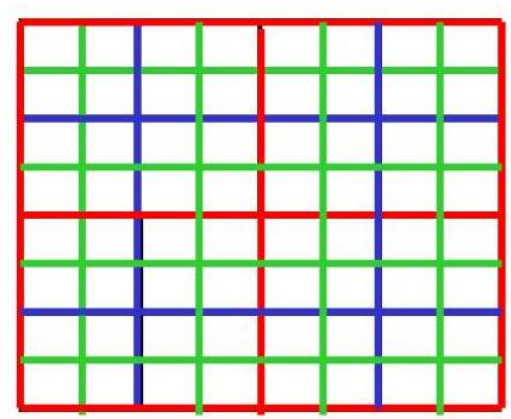

Fig. 1. Fugure $1-\boldsymbol{L}$-, $\boldsymbol{P}$-, and $\boldsymbol{G}$-superpixels in the image ( those corresponds to green, blue and red borders respectively)

- Local - the space defined by a pixel itself ( $\boldsymbol{L}$ superpixel );

- Proximal ( medium) - regions grouping several pixels - $\boldsymbol{L}$-superpixels - within their neighborhoods and producing $\boldsymbol{P}$-superpixels ;
- Global - further along the scale, a global region cover a much larger portion of the image square than a proximal region and grouping P-superpixels into $\boldsymbol{G}$-superpixels.

To the spatial scales $\boldsymbol{L}, \boldsymbol{P}$, and $\boldsymbol{G}$ correspond specific links among the pixels respectively just to connected regions of given scale. But contrary to [29 ] and [ 31], we utilize entity segmentation not to perform semantic labeling nor to reduce processing time but for adequate conversion of original images into complex networks.

For regular images, the classical Gestalt principles of grouping include proximity, similarity, common fate, good continuation, closure, symmetry, parallelism and are augmented by synchrony, common region, element and uniform connectedness) [ 32] In our pilot model, for partitioning image into superpixels we use simple grouping criteria with distance accent only and no concern to color intensity. The argument of this superpixel grouping is: pixels are not natural entities to follow any other criteria and these are merely the digital representation of images of given sizes and resolutions. Thus it is of sense to work with "superpixels' which bear not only local properties but those of medium and/or global. These preserve most of the specific traits at the scale of interest and in their links reflect natural processes of growing of network structures.

An image represents a bitmap, $\boldsymbol{N} \cdot \boldsymbol{M}, \boldsymbol{N}$ and $\boldsymbol{M}$ are the numbers of rows and columns respectively. We assume that in the network to be constructed, the number of nodes is equal to the number of superpixels in the partitioned image I . I.e. we define a one-to-one correspondence between node $v_{c i}(i=1, \ldots$

, , $\left.n_{c}\right), V_{L} \subset V_{P} \subset V_{G}$ and superpixel $s_{c i}\left(i=1, \ldots, n_{c}=N_{c} \cdot M_{c}\right)$

in pertinent category $\boldsymbol{c}=\{\boldsymbol{L}, \boldsymbol{P}, \boldsymbol{G}\}$.

For a given graph $G=\left(V_{L}, E\right)$ where each vertex in the set $\boldsymbol{V}$ is associated with an each node (superpixel in the image): vertices in $\boldsymbol{V}$ cover the image and the set $\boldsymbol{E}=\boldsymbol{E}_{\boldsymbol{L}} \boldsymbol{U} \boldsymbol{E}_{\boldsymbol{P}} \boldsymbol{U} \boldsymbol{E}_{\boldsymbol{G}}$ represents the edges, associated with links between pairs of $\boldsymbol{L}$ superpixels. Each pair of vertexes $\boldsymbol{e}_{c i j}\left(\boldsymbol{v}_{c i}, \boldsymbol{v}_{c j}\right)$ that corresponds the superpixels $\left(\boldsymbol{s}_{c i} ; \boldsymbol{s}_{c j}\right),\left(\boldsymbol{i}, \boldsymbol{j}=\mathbf{1}, \ldots, \boldsymbol{n}_{c} ; \boldsymbol{i} \neq \boldsymbol{j}\right)$ of category $c$ are connected through their "central" pixels or not connected by a link that depends on a proximity criteria with distance and color intensity components.

We followed $[23,24]$ to construct the components.

The spatial one is defined as Euclidean distance to assess deployment difference of two superpixels $\boldsymbol{i}$ and $\boldsymbol{j}$ as lattice nodes in 2-dimensional space:

$$
d_{e} \mathbb{R}\left(s \rrbracket_{c i}, s_{c j}\right)=\sqrt{\llbracket\left(s \rrbracket_{c i x}-s_{c j x}\right)^{2}+\mathbb{Z}\left(s \rrbracket_{c i y}-s_{c j y}\right)^{2}}
$$

To reflect gray-level intensity similarity between super pixels a color image is transformed into grey-level one in line with [23]:

$$
\mathrm{Y}=0.2126 \cdot \mathrm{r}+0.7152 \cdot \mathrm{g}+0.0722 \cdot \mathrm{b}
$$


Each superpixel of the original image comprises 8 bits of information that corresponds to 256 gray-level intensities.

To assign intensities to $\boldsymbol{P}$ - and $\boldsymbol{G}$ - superpixels averaging through all the inner superpixels is utilized:

$$
\begin{gathered}
Y_{P}=\left(\Sigma_{i=1}^{n_{L}} Y_{E i}\right) / n_{E} \\
Y_{G}=\left(\Sigma_{i=1}^{n_{P}} Y_{P i}\right) / n_{P}
\end{gathered}
$$

We claim pixels- nodes per se $\boldsymbol{s}_{\boldsymbol{P}}^{*}\left(\boldsymbol{s}_{\boldsymbol{P}}^{*}, \boldsymbol{s}_{\boldsymbol{P} y}^{*}\right)$ to be "central" in a $\boldsymbol{P}$-superpixel and $\boldsymbol{s}_{G}^{*}\left(\boldsymbol{s}_{G x}^{*}, \boldsymbol{s}_{G y}^{*}\right)$ to be "central" in a $\boldsymbol{G}$-superpixel thus with intensity of former to fit average one of the latter:

$$
Y\left(s_{P}^{*}\right)=Y_{P} \quad ; \quad Y\left(s_{G}^{*}\right)=Y_{G}
$$

Further, gray-level difference between the two superpixels $\boldsymbol{i}$ and $\boldsymbol{j}$ in category $\boldsymbol{c}$ is found by Manhattan distance: $\mid \boldsymbol{Y}\left(\boldsymbol{s}_{c i}\right)$ $\boldsymbol{Y}\left(\boldsymbol{s}_{c j}\right) \quad$.

So, the criteria to decide whether there is or not an edge between vertexes $\left(\boldsymbol{v}_{c i}, \boldsymbol{v}_{c j}\right)$ can be expressed as follows:

$$
\boldsymbol{e}_{c i j}\left(v_{c i}, v_{c j}\right) \exists \text {, if }
$$

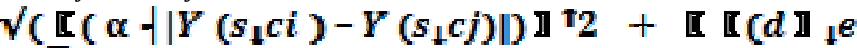 $\leq \boldsymbol{T}$, and $\mathbf{Z}$ otherwise.}

Here $\boldsymbol{T}$ is a threshold value ; $\boldsymbol{\alpha}, \boldsymbol{\beta}>\boldsymbol{0}$ are the parameters to balance contribution of intensity change and spatial distance to the criteria respectively.

Connectivity ( degree) distribution $\boldsymbol{N}(\boldsymbol{\kappa})$ was taken as a principal assessment metric for the resulting network graphs. We also calculate average connectivity (degree) for the converted network ( graph):

$$
\left\langle k>=\frac{1}{n} \sum_{i=1}^{n} k_{i}\right.
$$

where $\boldsymbol{k}$ is a connectivity ( degree ) of vertex $\boldsymbol{i}$, and average clustering coefficient:

$$
\left\langle C>=\frac{1}{n} \sum_{i=1}^{n} C_{i}\right.
$$

$$
C_{i}=\frac{2 m_{i}}{k_{i}\left(k_{i}-1\right)}, \quad \text { here } \boldsymbol{m}_{i} \text { is a number of links }
$$
between closest neighbors of vertex $\boldsymbol{i}$.

\section{INSTRUMENTS}

A specialized image-network convertor in the Python programming language supported by Igraph package has been created for network-aimed analyzing of an image and concomitant rendering of the network structure. (The standard Igraph package envisages merely graph creation [33].) Thus in addition to the standard Igraph functions, the convertor provides a possibility of formations of $\boldsymbol{L}-, \boldsymbol{P}$-, and $\boldsymbol{G}$ superpixels. All values - proximity thresholds , meso- and macro- superpixel sizes are controlled parameters of the convertor. Creation of plots was carried out by means of the Matplotlib tool [34].

\section{FINDINGS}

Case stydy

Two digital images have been taken to examine spatial scaled partition and conversion algorithm:

- manually prepared Black Annulus (BR) on white background Fig 2a;

- $\quad$ toucan picture (TP) [35 ], Fig. 2b

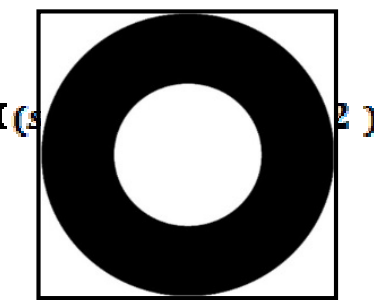

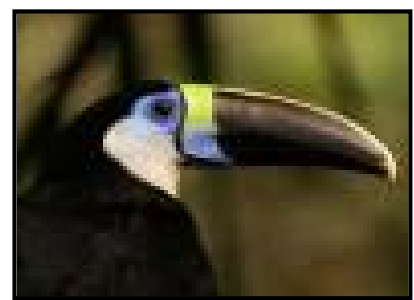

Fig. 2. Probe images: black annulus, BA (a) and toucan picture (TP)

BA had been previously compressed to $20 \times 20$ pixels and thus represents $n=400$ nodes in a pertinent converted network. Dimension of TP consists $96 \times 72$ pixels just to convert into 6912 nodes. The parameters $\boldsymbol{N}, \boldsymbol{M}, \boldsymbol{T}, \boldsymbol{\alpha}, \boldsymbol{\beta}$ and sizes of superpixels $\left|\boldsymbol{s}_{P}\right|$ and $\left|\boldsymbol{s}_{G}\right|$, have been varied .

Size of L-superpixel $\left|\boldsymbol{s}_{L}\right|=\mathbf{1}$.

\section{Resuls of converting}

According to the proposed methodology the BA and TP images were converted into complex The results are reflected

\begin{tabular}{|c|c|c|c|c|c|c|c|c|c|c|}
\hline \# Ima & $N$ & $M$ & $\mid \mathbf{s}_{L}$ & $\mid \boldsymbol{S}_{P}$ & $\left|\boldsymbol{s}_{G}\right|$ & $T$ & $\alpha$ & $\beta$ & $<\boldsymbol{k}>$ & $<C>$ \\
\hline $1 \mathrm{BA}$ & 20 & 20 & 1 & 5 & 10 & 0.5 & 1 & 1 & 292. & $0.868^{-}$ \\
\hline $2 \mathrm{BA}$ & 96 & 72 & 1 & 4 & 24 & 0.01 & 1 & 1 & 71.2 & 0.611 \\
\hline $3 \mathrm{BA}$ & 96 & 72 & 1 & 4 & 24 & 0.0075 & 1 & 1 & 38.3 & 0.583 \\
\hline $4 \mathrm{TP}$ & 96 & 72 & 1 & 4 & 24 & 0.015 & 1 & 1 & 7.92 & 0.493 \\
\hline $5 \mathrm{TP}$ & 96 & 72 & 1 & 4 & 24 & 0.015 & 1 & 1 & 19.5 & 0.553 \\
\hline $6 \mathrm{TP}$ & 96 & 72 & 1 & 4 & 24 & 0.015 & 1 & 0.1 & 7.92 & 0.493 \\
\hline
\end{tabular}
in the TABLE I with average values of connectivities and clustering coefficients and demonstrated by Fig. 3-8 presenting connectivity distributions and rendering network structures.

TABLE I. CALCULATED NETWORK METRICS FOR SEVERAL ADJUSTABLE PARAMETERS 


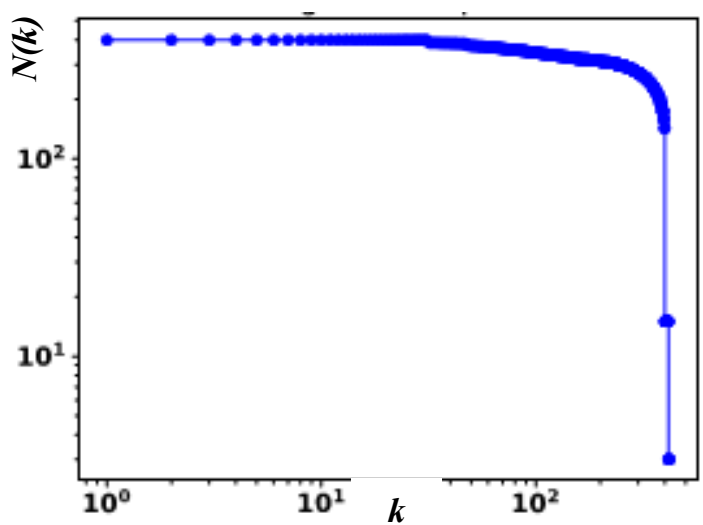

Fig. 3. Connectivity distribution : converted network (BA, \#1)

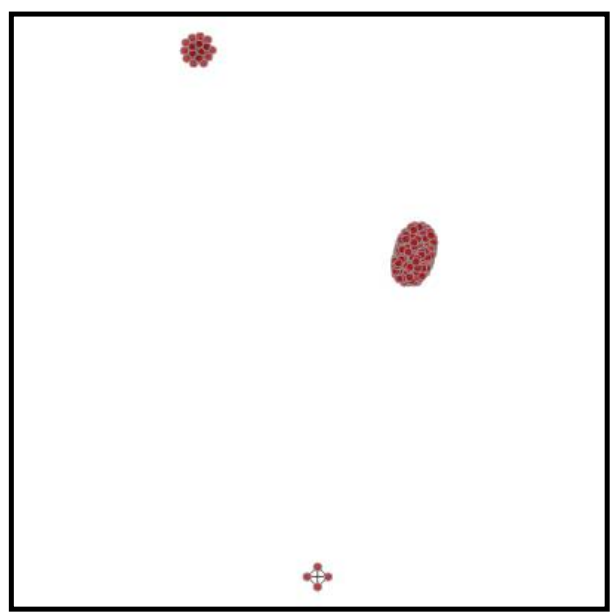

Fig. 4. Visualized converted network (BA, \#1)

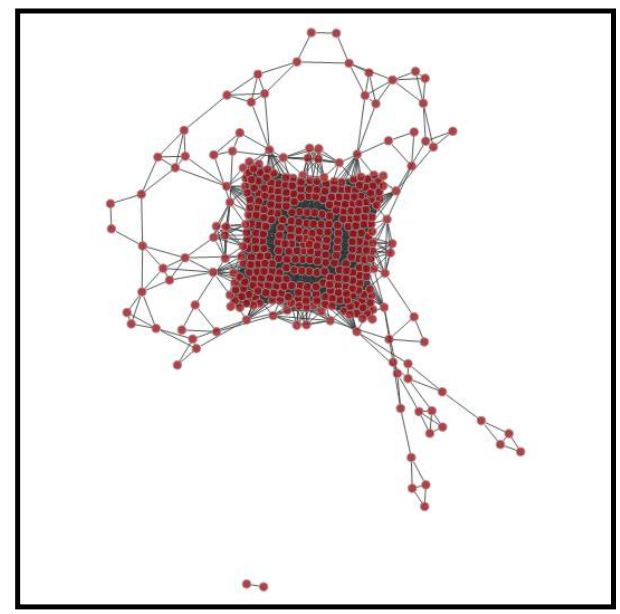

Fig. 5. Visualized converted network (BA, \#2)

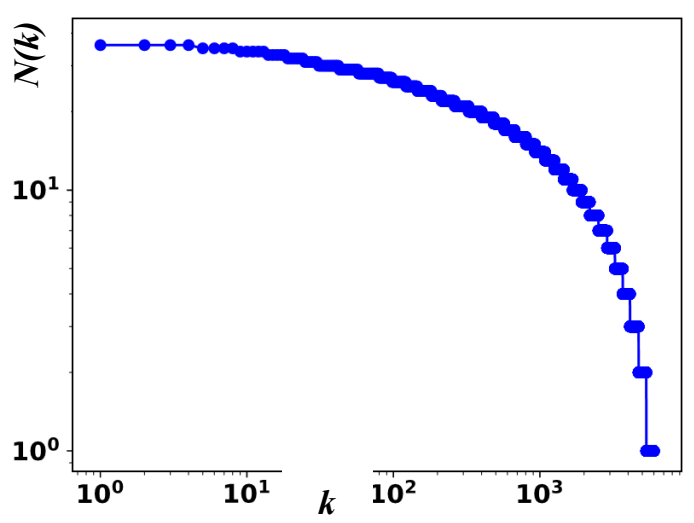

Fig. 6. Connectivity distribution for the converted network (TP, \#4)

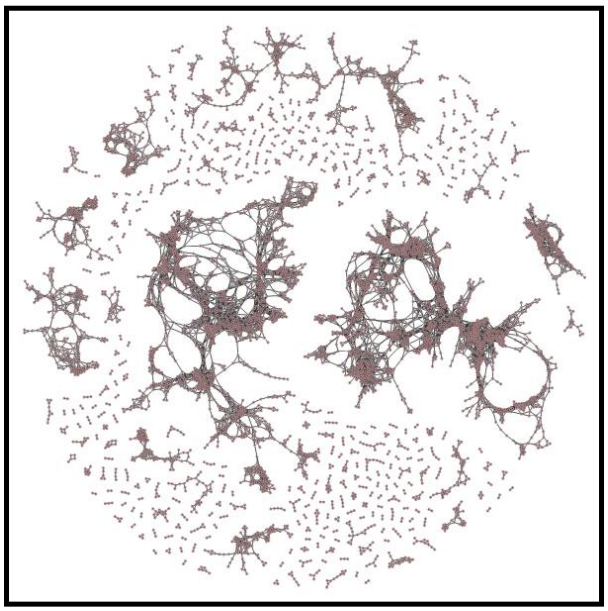

Fig. 7. Visualized converted network (TP, \#4 )

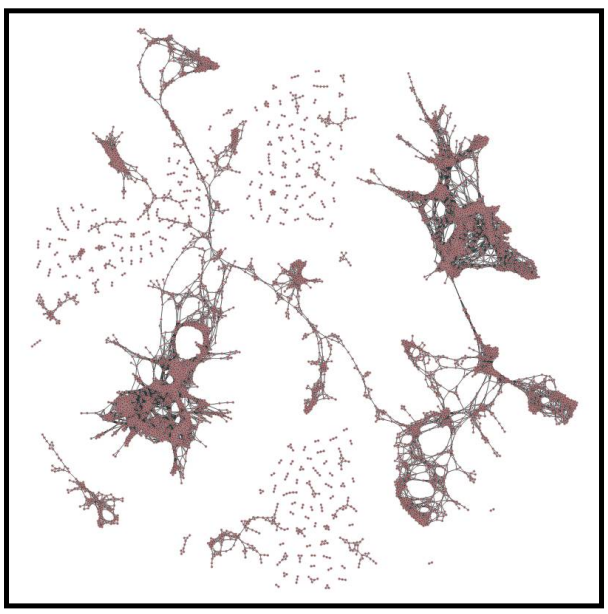

Fig. 8. Visualized converted network (TP, \#5)

The results show at which extent the converted networks are sensitive to the adjustable parameters. 


\section{CONCLUSIONS}

An approach to convert image data into genuine complex networks and further to assess topological properties of synthetic and real digital pictures has been proposed. This implies partition a multipixel image into 3 category levels which correspond to local, proximal, and global regions ( superpixels) and specific links among the pixels respectively to the connected regions.

Two probe pictures have been examined in line with rectangular superpixels to clarify how this new 3 spatial scale conversion into a complex network is growing and differ from that of traditional one. The results manifest sensitivity of final network structure to thresholds proximity of superpixels in distance and color intensity.

We suppose that global (macroscopic) and proximal (mesoscopic) views of a structured dataset, modeled as a network, will reveal more specific traits of the entity than a local-scale ( microscopic) one.

Some more steps to move forward with the new approach are envisaged such as sophisticated superpixel (non rectangular) segmentation [29] harmonized with scaledependent context and PCA application [25] for assessments of the complex networks.

\section{ACKNOWLEDGMENTS}

N.K. and A.T. thank Irkutsk Supercomputer Center of SB RAS ( Irkutsk, RF) for technical and organizational support in access to HPC-cluster "Academician Matrosov"[36].

\section{REFERENCES}

[1] A.-L.Barabasi, R. Albert. "Emergence of scaling in random networks" . Science. 286 (5439),1999, pp.509-512

[2] S.V.Buldyrev, R.Parshani, G.Paul, H.E.Stanely, S. Havlin.” Catastrophic cascade of failures in interdependent networks". Nature. 464, 2010, pp. 1025-1028

[3] B. Podobnik, D. Horvatić, M. Dickison, H. E. Stanley. "Preferential attachment in the interaction between dynamically generated interdependent networks". 2012. EPL (Europhysics Letters), 100( 5), 2012, 50004, 6 p.

[4] M.De Domenico. "Multilayer modeling and analysis of human brain networks" GigaScience ,6(5), 2017, pp. 1-8

[5] M. Kivela, A..Arenas, M.Barthelemy,.J. P. Gleeson, Y. Moreno, M.A.Porter " Multilayer networks". J.Complex Networks, 2(3), 2014, pp.203-271

[6] M. Bazzi, M. A. Porter, S. Williams, M. McDonald, D. J. Fenn, S. D. Howison, "Community detection in temporal multilayer networks, with an application to correlation networks," Multiscale Modeling \& Simulation,14(1),2016, pp. 1-41

[7] Z. Ashurova.,S. Myeong S., A. Tikhomirov, A.Trufanov , N.Kinash , O.Berestneva , A.Rossodivita. "Comprehensive Mega Network(CMN) Platform: Korea MTS Governance for CIS Case Study". Information Technologies in Science, Management, Social Sphere and Medicine (ITSMSSM 2016). Atlantis Press,2016,pp. 266 -269

[8] A.Majdandzic, B.Podobnik, S.V. Buldyrev, D.Y. Kenett, S. Havlin, H. E. Stanley. "Spontaneous recovery in dynamical networks". Nature Physics, (10), 2014, pp. 34-38

[9] T. C. Silva, L.Zhao . "Machine Learning in Complex Networks". Springer International Publishing .2016, 331 p.

[10] G. S. Domingues, F. N. Silva, C. H. Comin, L. da F. Costa "Topological characterization of world cities", arXiv:1709.08244v2 [physics.soc-ph], 2017, 11 p. URL: https://arxiv.org/pdf/1709.08244.pdf
[11] B.Podobnik , D. Horvatic, T. Lipic, M. Perc, J.M. Buldu, H.E. Stanley. "The cost of attack in competing networks". J. R. Soc. Interface (12), 2015, 20150770, 8 p.

[12] J. M. Montoya, S. L. Pimm , R. V. Sole . "Ecological networks and their fragility". Nature, 442(20), 2006, pp. 259-264

[13] V. Miele, C. Matias. " Revealing the hidden structure of dynamic ecological networks". Soc Open Sci, 4(6), 2017: 170251, 10 p.

[14] R. Milo, S. Shen-Orr, S. Itzkovitz, N. Kashtan, D. Chklovskii, U. Alon. "Network Motifs: Simple Building Blocks of Complex Networks". Science, 298, ( 5594) , 2002, pp. 824-827

[15] A.-L. Barabasi, N. Gulbahce, and J. Loscalzo, "Network medicine: a network-based approach to human disease," Nat. Rev. Genet., 12(1), 2011, pp. 56-68

[16] D. Trpevski, Wallace K. S. Tang, L. Kocarev. "Model for rumor spreading over networks". Phys. Rev. E, 81(5), 2010: 056102, 11 p.

[17] B. S. Khan , M. A. Niazi . " Emerging Topics in Internet Technology: A Complex Networks Approach “. arXiv:1708.00578, 2017, 5 p.

[18] S. Ferretti . "Clustering of Musical Pieces through Complex Networks: an Assessment over Guitar Solos". arXiv:1709.05193. 2017, 7 p.

[19] F. Pablo-Martí, A. Sánchez. "Improving transportation networks:Effects of population structure and decision making policies". Sci. Rep., 7 , $2017: 4498,9$ p.

[20] A.Karduni, A. Kermanshah , S. Derrible . "A protocol to convert spatial polyline data to network formats and applications to world urban road networks". Scientific Data. 3, 2016:160046, 7 p.

[21] X. Han, Z. Shen, W.-X. Wang, Z. Di. "Robust Reconstruction of Complex Networks from Sparse Data“. Phys. Rev. Lett. 114(2), 2015: 028701, $5 \mathrm{p}$.

[22] L.Lacasa, B. Luque, F. Ballesteros, J. Luque, J. C. Nuno . "From time series to complex networks: The visibility graph". Proc. Natl. Acad. Sci. USA ,105, (13), 2008, pp. 4972-4975

[23] M. Semenov, K. Lelushkina. "Study of the Materials Microstructure using Topological Properties of Complex Networks". IOP Conf. Series: Materials Science and Engineering, 135, 2016 : 012040, 4 p.

[24] Wu, Z., Lu, X. and Deng, Y. "Image edge detection based on local dimension: a complex networks approach”. Physica: A, 440 (1), 2015, pp. $9-18$

[25] G. V. L. de Lima, T.R. Castilho, P.H. Bugatti, P. T. M. Saito, F. M. Lopes. "A Complex Network-Based Approach to the Analysis and Classification of Images". In Proc. of 20th Iberoamer. Congress, CIARP : Progress in Pattern Recognition, Image Analysis, Computer Vision, and Applications. A. Pardo, J. Kittler (Eds.) ,2015, pp 322-330

[26] H. F. de Arruda, F. N. Silva, V.Q. Marinho, D. R. Amancio , L. da Fontoura Costa. "Representation of texts as complex networks: a mesoscopic approach". arXiv:1606.09636v2 [cs.CL], 2017, 14 p. URL: https://arxiv.org/pdf/1606.09636.pdf

[27] X. Ren , J. Malik, "Learning a classification model for segmentation," in Computer Vision, 2003. Proceedings. Ninth IEEE International Conference on. IEEE, 2003, pp. 10-17

[28] Y. Zhang, X. Li, X. Gao, C. Zhang. “ A simple algorithm of superpixel segmentation with boundary constraint “. IEEE Trans. Circuits and Systems for Video Techn. 27 (7), 2017, pp. 1502-1514

[29] O. Cuadros, G. Botelho, F. Rodrigues, J. B. Neto "Segmentation of Large Images with Complex Networks". 25th SIBGRAPI Conf. on Graphics, Patterns and Images., 2012, pp. 24 - 31 URL: https://pdfs.semanticscholar.org/f1ae/a7b84f6fb573d39075d9efcfe6e198 fd349b.pdf

[30] A.Aksac, T. Ozyer, R. Alhajj. "Complex Networks Driven Salient Region Detection based on Superpixel Segmentation", Pattern Recognition, 66 ( C), 2017, pp. 268-279

[31] M. Mostajabi, P.Yadollahpour, G.Shakhnarovich. "Feedforward semantic segmentation with zoom-out features." In: Proc. IEEE Conf. on Computer Vision and Pattern Recognition. 2015, pp. 3376-3385

[32] J.Wagemans , J.H.Elder, M.Kubovy , S.E. Palmer, M.A.Peterson , M.Singh, R. von der Heydt. "A century of Gestalt psychology in visual perception: I. Perceptual grouping and figure-ground organization". Psychol. Bull.,138(6), 2012, pp. 1172-217 
[33] G. Csardi, T. Nepuzs. "The igraph software package for complex network research". Intern. J. Complex. Syst. 1695, 2006, pp. 1-9

[34] J.D.Hunter. “ Matplotlib: 2-D grapghics environment”. IEEE Comp. Soc., 9(3), 2007, pp.90-95
[35] URL:https://pmdvod.nationalgeographic.com/NG_Video_DEV/935/ 811 /toucan $480 \times 360 . j p g$

[36] URL: http://hpc.icc.ru 